\title{
Wasser - elementare und strategische Ressource des 21. Jahrhunderts
}

\author{
I. Eine Bestandsaufnahme
}

\author{
Helmut Lehn · Oliver Parodi
}

Eingegangen: 30. September 2008/Akzeptiert: 28. Februar 2009/Online veröffentlicht: 27. März 2009

(C) Springer-Verlag 2009

Präambel Vor dem Hintergrund der wachsenden Weltbevölkerung, dem wirtschaftlichen Wachstum - gerade in Schwellenländern wie China und Indien - der globalen Vernetzung, dem weltweiten Klimawandel und der zunehmenden Umweltbeanspruchung stellt der Umgang mit begrenzten natürlichen Ressourcen eine gewaltige Herausforderung dar. Dies betrifft auch und insbesondere die Ressource Wasser, die wie kein anderer Naturstoff in alle menschlichen Lebensbereiche einverwoben ist. Aus diesem Grunde gilt Wasser als eine elementare Ressource und der Zugang zu ihr als strategisches Anliegen von Gesellschaften. Nutzungs- und Interessenkonflikte führen immer wieder zu sozialen Spannungen und politischen Konflikten. Nicht selten werden für die Zukunft gar Kriege um Wasser vorhergesagt. In drei Beiträgen werden Hintergründe einer konstatierten ,globalen Wasserkrise“ aufgearbeitet und Vorschläge eines nachhaltigen Umgangs mit Wasserressourcen skizziert.

Teil I. Eine Bestandsaufnahme: Vorkommen und Verfügbarkeit der Ressource Wasser werden vor dem Hintergrund globaler Wasserkreisläufe diskutiert.

Teil II. Wasser im Nutzungskontext: Dieser Artikel widmet sich der menschlichen Nutzung und Inanspruchnahme von Wasser und weist Problemlagen aus.

Teil III. Ansätze nachhaltiger Inanspruchnahme: Abschließend werden strategische Defizite und Lösungswege im Umgang mit Wasser aufgezeigt.

Verantwortlicher Herausgeber: Henner Hollert

H. Lehn $(\bowtie) \cdot$ O. Parodi

ITAS - Institut für Technikfolgenabschätzung und Systemanalyse,

FZK - Forschungszentrum Karlsruhe $\mathrm{GmbH}$,

Hermann-von-Helmholtz-Platz 1,

76344 Eggenstein-Leopoldshafen, Deutschland

E-Mail: helmut.lehn@itas.fzk.de, oliver.parodi@itas.fzk.de
Zusammenfassung Hintergrund und Ziel Die steigende Nachfrage nach Süßwasser bei begrenzten oder als Folge des Klimawandels regional zurückgehenden Wasserressourcen, verknüpft mit einer zunehmenden qualitativen Belastung, wirft immer häufiger die Frage auf, ob die Bevölkerung einzelner Regionen, ganzer Kontinente oder gar der Erde in Zukunft mit einer Mangelsituation bezüglich verfügbarer Wasserressourcen konfrontiert sein wird.

Diskussion Aufbauend auf einer Analyse der drei global relevanten Wasserkreisläufe (mariner, terrestrischer und Verbindungskreislauf) werden die einwohnerspezifischen sich erneuernden Wasserressourcen der Kontinente quantitativ abgeschätzt. Die aufgrund technisch-ökonomischer Restriktionen zugänglichen Wassermengen werden den menschlichen Nutzungsansprüchen gegenübergestellt und dabei die herausragende Bedeutung der Erzeugung von Biomasse (zur Verwendung als Nahrungsmittel, Futtermittel, Produktionsstoff oder Energieträger) im Hinblick auf den weltweiten Bedarf von Wasser dargestellt.

Ergebnisse Im globalen Maßstab besteht kein Wassermangel. Knappheiten treten regional oder saisonal auf. Ein Großteil des weltweit eingesetzten Wassers dient zur Erzeugung von Biomasse. Deshalb liegt in diesem Sektor ein wesentlicher Schlüssel zum strategischen Umgang mit Wasser. Die bisher übliche Bilanzierung von Wasserdargebot und -nachfrage übersieht oftmals auf der Dargebotsseite die Bedeutung des so genannten ,grünen Wassers" bei der Erzeugung von Biomasse und suggeriert deshalb Knappheiten, die de facto so nicht bestehen. Unserer Ansicht nach ist die Mengenangabe des sich jährlich global erneuernden Süßwasservorkommens im Hinblick auf die gesamte menschliche Wassernutzung deutlich $\mathrm{zu}$ korrigieren. Die in der heutigen Literatur dominierende Angabe von etwa $40.000 \mathrm{~km}^{3}$ ist auf über $100.000 \mathrm{~km}^{3}$ anzuheben. 
Schlussfolgerungen und Perspektiven Wassermangel besteht weniger aus Gründen mangelnder Ressourcenverfügbarkeit oder aufgrund fehlender Technologien, sondern oftmals mangels Bewusstseins, fehlenden politischen Willens oder unzureichenden sozio-ökonomischen Potenzialen. Aufgrund einer umfassenden Dargebots-Nachfrage-Bilanzierung, die alle Wassernutzungsformen integriert, sollten nationale und internationale Potenziale für die Bedarfsdeckung analysiert werden, um darauf aufbauend notwendige Versorgungsszenarien zu entwickeln.

Schlüsselwörter Biomasse · Blaues Wasser · Grünes Wasser · Wasser · Wasserdargebot · Wasserkreislauf · Wasserressourcen · Wasserverfügbarkeit

\section{Water - fundamental and strategic resource of the 21st century}

\begin{abstract}
Background and aim The combination of increasing freshwater demand, limited or regionally decreasing water resources as a consequence of the climate change, and an increasing qualitative burden, lead more and more to the question as to whether the population of particular regions, whole continents or even the entire world will be confronted with a shortage of available water resources in the future.

Discussion The resident-specific regenerative water resources of the continents are being quantitatively rated on the basis of an analysis of the three globally relevant water cycles (the marine one, the terrestrial one and the one which is linking both of them). The available amount of water is limited by technical-economical restrictions. This amount is compared with human demands, and the outstanding relevance of the production of biomass (to be used as food, animal feed, primary product or energy carrier) regarding the worldwide water need is discussed.

Results From a global point of view there is no water shortage. Scarcities occur only regionally or seasonally. A large portion of the water employed worldwide is used for the production of biomass. Therefore, this sector provides a considerable key to the strategic handling of water. The socalled 'green water' is crucial for the production of biomass. However, the common way of balancing water supply and demand often overlooks the importance of the green water on the supply side and thus indicates shortages which de facto do not exist. As to the whole usage of water by humans, in our opinion, the stated quantity of freshwater which is being regenerated every year all over the world has to be corrected considerably. The figure of $40,000 \mathrm{~km}^{3}$ which is often quoted in current literature has to be raised to more than $100,000 \mathrm{~km}^{3}$.

Conclusions and perspectives Water shortage is often not a result of limited resources or technologies but is a con-
\end{abstract}

sequence of missing awareness, failing political volition or insufficient socio-economic potentials. On the basis of a comprehensive water supply/demand balance, which includes all forms of water usage, national and international potentials for the fulfilment of the demand should be analysed in order to be able to develop necessary supply scenarios.

Keywords Biomass · Blue Water - Green Water · Water · Water Supply · Water Cycle · Water Resources · Water Availability

\section{Problemstellung}

Seit einigen Jahren gerät das Thema „Wasser“ zunehmend ins mediale Rampenlicht und auf die Tagesordnungen politischer Debatten und Programme. „Umgang mit Wasserknappheit", so lautete beispielsweise das Motto des Weltwassertags der Vereinten Nationen im Jahr 2007 (UNESCO 2007). Im Jahr 2005, dem Startjahr der Internationalen UNDekade „Water for Life“ fand in Hamburg der Kongress statt: „Warnsignal Klima: Genug Wasser für alle“ (Lozán et al. 2005). ,Wasser - Konfliktstoff des 21. Jahrhunderts?“ lautete das Motto einer Konferenz im Sommer 2007 (HAW 2007). Diese Ereignisse stehen hier stellvertretend für eine immer größer werdende Sorge um den globalen Umgang mit Wasser als Ressource. ${ }^{1}$

Die elementare Bedeutung von Wasser für „Natur“ und Zivilisation ist hinlänglich bekannt: Ohne Wasser kein Leben, keine Zivilisation, kein Wohlstand, kein Glück. Als fundamentale Existenzbasis sowie als unersetzbarer Stoff vieler natürlicher und technischer Produktionsprozesse wird Wasser zu einer elementaren Ressource.

Die heutige mitteleuropäische Normalität einer stets gesicherten Wasserversorgung und -entsorgung lässt gerne in Vergessenheit geraten, dass in anderen Regionen der Erde massive Wasserprobleme zu beklagen sind. Über eine Milliarde Menschen haben weltweit keinen adäquaten Zugang zu Wasser. Mehr als doppelt so viele Menschen, ein Drittel der Erdbevölkerung, müssen ohne sanitäre Einrichtungen leben. Etwa 5.000 Kinder sterben täglich (1,8 Mio. jährlich) an wassergebundenen Krankheiten (Borrazzo und Rainey 2007). In Entwicklungs- und Schwellenländern sind mit einer ungenügenden Bewirtschaftung der Wasserressourcen vielfach Migration, Vertreibung, Verarmung, Repressionen und ökologische Defizite verbunden. Sei es, dass dort bereits wasserwirtschaftliche Defizite wie Wasserarmut oder -verschmutzung bestehen, sei es, dass aktuelle unausgewogene wasserwirtschaftliche Projekte, wie

\footnotetext{
${ }^{1}$ Fragen des Wasserüberschusses (Überschwemmungen, Meeresspiegelanstieg) sind nicht Gegenstand dieses Beitrags.
} 
z. B. der Bau großer Staudämme oder Landnutzungsänderungen, soziale und ökologische Verwerfungen erst nach sich ziehen.

Auch hierzulande sind mit Wassernutzungen problematische Folgen verbunden. Man denke z.B. an Arzneimittelrückstände und endokrine Stoffe in Oberflächen- und Grundwasser (Kern und Lorenz 2008; Püttmann et al. 2008; Kratz et al. 2000), lokale Übernutzung von Wasservorkommen (vgl. Froebrich et al. 2007; Crosa et al. 2006), extraterritoriale Wassernutzung (s. Teil II) oder ökologische Defizite. Bevölkerungswachstum, Klimawandel (Stock 2003), globales Wirtschaftswachstum und globale Handelsströme üben bereits heute einen starken Einfluss auf die lokale und regionale Verfügbarkeit von Wasser aus und werden bestehende Wasserproblematiken vielfach noch verschärfen oder neu erzeugen.

Zur strategischen Ressource wird Wasser letztlich aufgrund seiner elementaren Bedeutung und der auf der Erde naturgegebenen Beschränktheit und Ungleichverteilung seines Vorkommens in zeitlicher und räumlicher Dimension. So war es auch in der Geschichte der Menschheit eine der ersten großtechnischen Unternehmungen und zivilisatorischen Leistungen, diese naturgegebene Ungleichverteilung mittels wasserbaulicher Anlagen auszugleichen, Wasser zu speichern und es über Distanzen hinweg verfügbar zu machen. Nicht zuletzt die Entstehung nahezu aller frühen Hochkulturen stand in Zusammenhang mit einem erfolgreichen Wassermanagement im groß angelegten Maßstab (Wittfogel 1962).

Auch heute ist der Umgang mit Wasser oft ein im weiten Sinne strategisches Anliegen, nämlich das eines langfristig angelegten, zielgerichteten und planvollen Handelns. Auf nationaler Ebene sei hier an erster Stelle die in vielen Staaten institutionalisierte Wasserwirtschaftsplanung genannt. Auf internationaler Ebene findet ein strategischer Umgang mit der Ressource Wasser z. B. in der Arbeit grenzüberschreitender Flussgebietskommissionen oder in Regelwerken multilateraler Verträge zwischen Flussanrainerstaaten statt. ${ }^{2}$ Diese sollen Gefahren abwenden und einer Übernutzung der Ressource dauerhaft vorbeugen. Hierbei gilt es, die Interessen einzelner Nutzer auszugleichen, ein gemeinsames Wohl zu fördern und Nutzungskonflikte zu vermeiden bzw. diese sozialverträglich beizulegen. Konflikte um die Nutzung von Wasser treten dabei sowohl territorial auf (z. B. zwischen Gewässeranrainern - wie bspw. Sudan und Ägypten, Türkei und Syrien) oder sektorial (z. B. zwischen Landwirtschaft, Industrie, Tourismus) wie auch ent-

\footnotetext{
${ }^{2}$ Beispiele hierfür sind die Internationale Kommission zum Schutze des Rheins gegen Verunreinigungen [IKSR], die seit 1957 aus Vietnam, Laos, Kambodscha und Thailand bestehende Mekong Kommission, die Initiative für das Einzugsgebiet des Nils [NBI] (Dawoud 2001) oder auch das Umweltabkommen zwischen dem Hafen Rotterdam und dem Verband der deutschen Chemischen Industrie (Lehn et al. 1996).
}

lang von „Klassengrenzen“ (z. B. arm/reich, hoch/gering technisiert). ${ }^{3}$

Aufgrund seiner für Individuen und Volkswirtschaften (Staaten) überlebenswichtigen Funktionen kann Wasser letztlich auch zum Gegenstand eines im engen, machtpolitischen Sinne „strategischen“ Handelns werden. Historisch sind etliche Konfliktfälle um (gemeinsame) Wassernutzungen sowohl mit der politischen Androhung von Gewalt als auch mit gewalttätigen Auseinandersetzungen dokumentiert. Unter den ca. 1800 Zwischenfällen, die sich im letzten halben Jahrhundert um die Nutzung von Wasserressourcen ereignet haben, lassen sich jedoch keine ausgesprochenen „Wasserkriege“ belegen (Wolf 2001; Süddeutsche Zeitung 2007). Allerdings werden immer wieder Stimmen prominenter Akteure laut, die Kriege um Wasser prophezeien: „If the wars of the twentieth century were fought over oil, the wars of this century will be fought over water." (The World Bank, in: Clarke and King 2004).

Ein Ziel dieser Beitragsserie ist es auch, solche Prophezeiungen kritisch zu hinterfragen und Wege zu einem strategischen Umgang mit Wasser aufzuzeigen, der sich an den Zielen einer nachhaltigen Entwicklung orientiert. Zunächst gilt es, eine Bestandsaufnahme über Angebot und Nachfrage nach Süßwasser zu liefern. Durch die elementare Bedeutung von Wasser und seine dementsprechend mannigfaltigen Verflechtungen mit Öko- und Wirtschaftssystemen sowie seine physische Mobilität ist dies kein triviales Unterfangen.

Um nun sowohl dieser Komplexität Rechnung zu tragen als auch die Darstellung handhabbar zu halten, wird „Wasser“ im Folgenden unter drei Perspektiven beleuchtet: Erstens unter ökosystemaren Aspekten (z. B. Wasserkreislauf, Wasserdargebot), zweitens unter einem technischökonomischen Blickwinkel (Wasserförderung, Nutzung, Inanspruchnahme $-\mathrm{s}$. Teil II), der bereits Problemlagen und Konfliktpotenziale erkennen lässt. Eine dritte umfassende Perspektive führt - unter Berücksichtigung politischer, ökonomischer und sozialer Aspekte - die beiden vorangegangenen zusammen und zeigt Vorschläge für einen nachhaltigen Umgang mit der Ressource Wasser auf (s. Teil III).

\section{Wasserdargebot}

Unsere Überlegungen zum Umgang mit Wasser nehmen ihren Ausgangspunkt in der zur Verfügung stehenden Wassermenge. Der gesamte Wasservorrat unseres „blauen“ Planeten wird auf 1.400 Mio. $\mathrm{km}^{3}$ geschätzt. Davon bestehen nur 2,5\% oder 35 Mio. $\mathrm{km}^{3}$ aus Süßwasser. Etwa zwei Drittel

\footnotetext{
${ }^{3}$ In Entwicklungsländern kommt es oftmals zu existenziellen Wassernutzungskonflikten zwischen exportorientierter Agrarindustrie und Subsistenzwirtschaft der armen, meist indigenen Bevölkerung (z.B. der Konflikt um Holzressourcen der Mapuche in Chile, s. Völpel 2008).
} 
Abb. 1 Der globale Wasserkreislauf; Angaben in $1.000 \mathrm{~km}^{3}$ (verändert nach Lemke 2002)

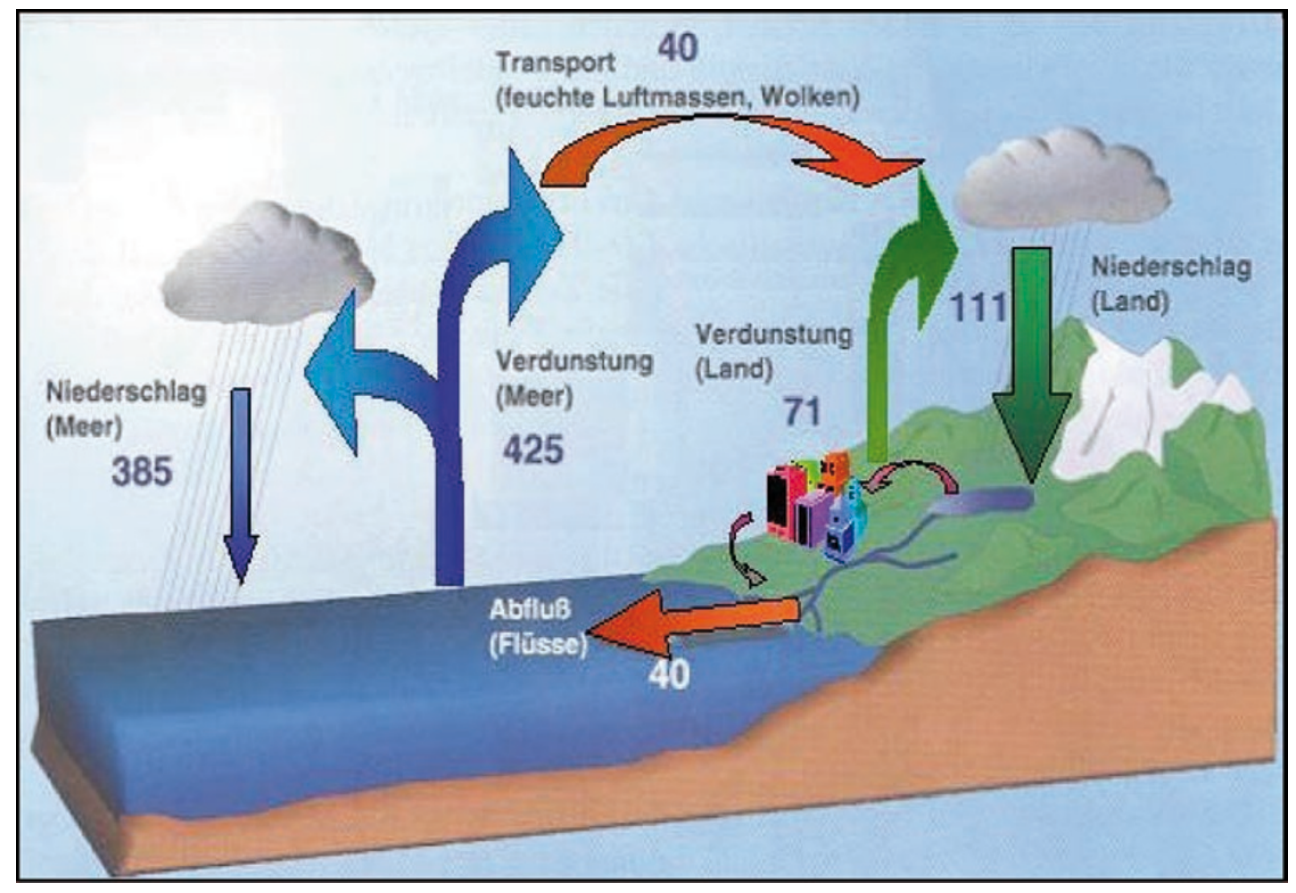

hiervon sind im Eis von Gletschern und Polkappen festgelegt, rund 30\% liegen als Grundwasser vor. Weniger als $1 \%$ des Süßwassers (ca. 0,1 Mio. $\mathrm{km}^{3}$ ) ist in Flüssen und Seen enthalten (Dyck und Peschke 1983; Lozán et al. 2005).

\subsection{Die globale Ressource Wasser und ihre Regeneration}

Wasser ist eingebunden in Kreisläufe. Angetrieben von der Sonnenenergie verdunsten im langjährigen Mittel jährlich ca. $425.000 \mathrm{~km}^{3}$ Wasser über den Ozeanen. $385.000 \mathrm{~km}^{3}$ (ca. $90 \%$ ) regnen sich wieder über ihnen ab (mariner Wasserkreislauf, grau in Abb. 1, siehe Titelbild). Lediglich ca.10\% oder $40.000 \mathrm{~km}^{3}$ erreichen das Festland der Kontinente (Verbindungskreislauf). Zusammen mit den dort verdunsteten ca. $71.000 \mathrm{~km}^{3}$ entsteht insgesamt eine kontinentale Niederschlagsmenge von etwa $111.000 \mathrm{~km}^{3}$ (Lemke 2002). ${ }^{4}$ Etwa sechs Prozent des Niederschlags, $6.000 \mathrm{~km}^{3}$, verdunsten wieder von Wasser- und Bodenoberflächen (Evaporation), mehr als das Zehnfache, nämlich ca. $65.000 \mathrm{~km}^{3}$ (Lozán et al. 2005; Zehnder 2002), werden durch die grünen Pflanzen in die Atmosphäre abgegeben (Transpiration). Beide Prozesse, die unter dem Begriff Evapotranspiration zusammengefasst werden, bilden zusammen mit dem Niederschlag den terrestrischen Wasserkreislauf (grün in Abb. 1, siehe Titelbild). Der Rest, ca. $40.000 \mathrm{~km}^{3}$, fließt zu zwei Drittel mit den Flüssen, zu einem Drittel unterirdisch als Grundwasser in die Ozeane

\footnotetext{
${ }^{4}$ Die Zahlenangaben differieren in der Literatur um etwa $15 \%$ : So beziffert Zehnder (2002) den kontinentalen Niederschlag im Mittel mit $113.000 \mathrm{~km}^{3}$ - bei einer Spannbreite von $90.000 \mathrm{~km}^{3}$ bis $119.000 \mathrm{~km}^{3}$.
}

zurück (Zehnder 2002), wodurch sich der Verbindungskreislauf zwischen Ozeanen und dem Festland schließt (blau in Abb. 1, siehe Titelbild). Diese zwischen den Ozeanen und dem Festland ,pendelnden“ etwa $40.000 \mathrm{~km}^{3} / \mathrm{a}$ werden in der Literatur als die sich jährlich erneuernde globale Süßwasserressource angesehen. ${ }^{5}$ Diese klassische Betrachtungsweise wird in Kapitel 4 noch kritisch zu hinterfragen sein.

Geht man aber zunächst einmal, wie üblich, von $40.000 \mathrm{~km}^{3}$ aus, so ergibt sich bei einer Weltbevölkerung von derzeit ca. 6,7 Mrd. Menschen (Deutsche Stiftung Weltbevölkerung 2008) eine Erneuerungsrate von ca. $6.000 \mathrm{~m}^{3} \mathrm{Süß-}$ wasser pro Einwohner und Jahr im globalen Durchschnitt.

Je nach betrachtetem Zeitraum erscheinen größere oder geringere Wassermengen als Bestandteile des globalen Wasserkreislaufs. Dies liegt in der Varianz der Dynamik unterschiedlicher Wassermassen begründet. Wasser der Atmosphäre ist ungleich mobiler als Wasser aus tiefen Gesteinsschichten. Die durchschnittlichen Erneuerungszeiträume reichen von 8 Tagen (Wasser in der Atmosphäre) über 17 Tage (Flusswasser), wenige Jahre bis Jahrzehnte (Grundwasser) bis zu mehren tausend Jahren (Ozeane, tiefes Grundwasser, Gletscher) (Lozán et al. 2005). Die quantitativen Regenerationszeiten unterschiedlicher Wassermassen unterscheiden sich also bis um den Faktor 100.000.

Dies lässt deutlich werden, dass für die Ressource „Wasser“ die übliche idealtypische Unterscheidung in ,regene-

\footnotetext{
${ }^{5}$ Z. B. Gleick 2000; Lemke 2002; Lozán et al. 2005; World Resources Institute 2007. Die Angaben in der Literatur schwanken hier zwischen 40.000 und $47.000 \mathrm{~km}^{3}$.
} 
rativ“ oder „nicht-regenerativ“ pauschal nicht greift. Angemessener wäre es, die dichotome Einteilung regenerativ/ nicht-regenerativ zu Gunsten einer skalierten Betrachtung aufzugeben, bei der die Regenerationszeiträume kontextbezogen (örtlich, situativ) den menschlichen Nutzungen gegenübergestellt werden.

\subsection{Regionale Disparitäten}

Wasser ist eine regionale und mobile, ,flüchtige“ Ressource. Je nach Ort und Zeit differieren die Wasservorkommen sehr stark. Betrachtet man beispielsweise die Verteilung des durchschnittlich jährlichen Wasserdargebots auf kontinentaler Ebene und setzt dieses ins Verhältnis zur jeweiligen Bevölkerung, so erkennt man erhebliche Unterschiede bzgl. der spezifischen Verfügbarkeit (Tabelle 1; vgl. Gleick 2000).

So verfügt z. B. Australien pro Einwohner über zwanzigmal mehr Wasser als Asien. In Südamerika erneuert sich pro Kopf über neunmal mehr Wasser als in Europa. Bezogen auf die Anzahl der Einwohner ist die Erneuerungsrate von Süßwasser in Afrika, Asien und Europa geringer als im globalen Durchschnitt, während sie in Amerika und Australien weit darüber liegt.

Diese kontinentale Ressourcenverteilung sagt indes wenig über die regionale oder lokale Verfügbarkeit von Wasser
Tabelle 1 Kontinentale Wasserverfügbarkeit im Jahr 1995 (Daten nach Gleick 2000)

\begin{tabular}{|c|c|c|c|}
\hline Kontinent & $\begin{array}{l}\text { Erneuerbare } \\
\text { Wasserressource } \\
\text { absolut }\left(\mathbf{k m}^{3} / \mathbf{a}\right)\end{array}$ & $\begin{array}{l}\text { Bevöl- } \\
\text { kerung } \\
\text { (Mio.) }\end{array}$ & $\begin{array}{l}\text { Erneuerbare } \\
\text { Wasserres. pro } \\
\text { Kopf }\left(\mathbf{m}^{3} / \text { Ea) }\right.\end{array}$ \\
\hline Afrika & 4.050 & 708 & 5.720 \\
\hline Asien & 13.510 & 3.445 & 3.920 \\
\hline $\begin{array}{l}\text { Australien und } \\
\text { Ozeanien }\end{array}$ & 2.360 & 29 & 82.200 \\
\hline Europa & 2.900 & 685 & 4.230 \\
\hline $\begin{array}{l}\text { Nord- und Mittel- } \\
\text { amerika }\end{array}$ & 7.890 & 453 & 17.400 \\
\hline Südamerika & 12.030 & 315 & 38.200 \\
\hline $\begin{array}{l}\text { Alle Kontinente } \\
\text { (ohne Antarktis) }\end{array}$ & 42.740 & 5.635 & 7.580 \\
\hline
\end{tabular}

aus. Abbildung 2 stellt nun die erneuerbare Süßwasserressource pro Kopf für ausgewählte Staaten Europas und des Nahen Ostens dar. Innerhalb Europas bestehen beispielsweise zwischen Deutschland und Norwegen Unterschiede von mehr als dem 40-fachen. Im Nahen Osten beträgt die Erneuerungsrate von Süßwasser pro Einwohner weniger als ein Zehntel des europäischen Durchschnitts, was den dortigen Wassermangel deutlich illustriert. Insgesamt lassen

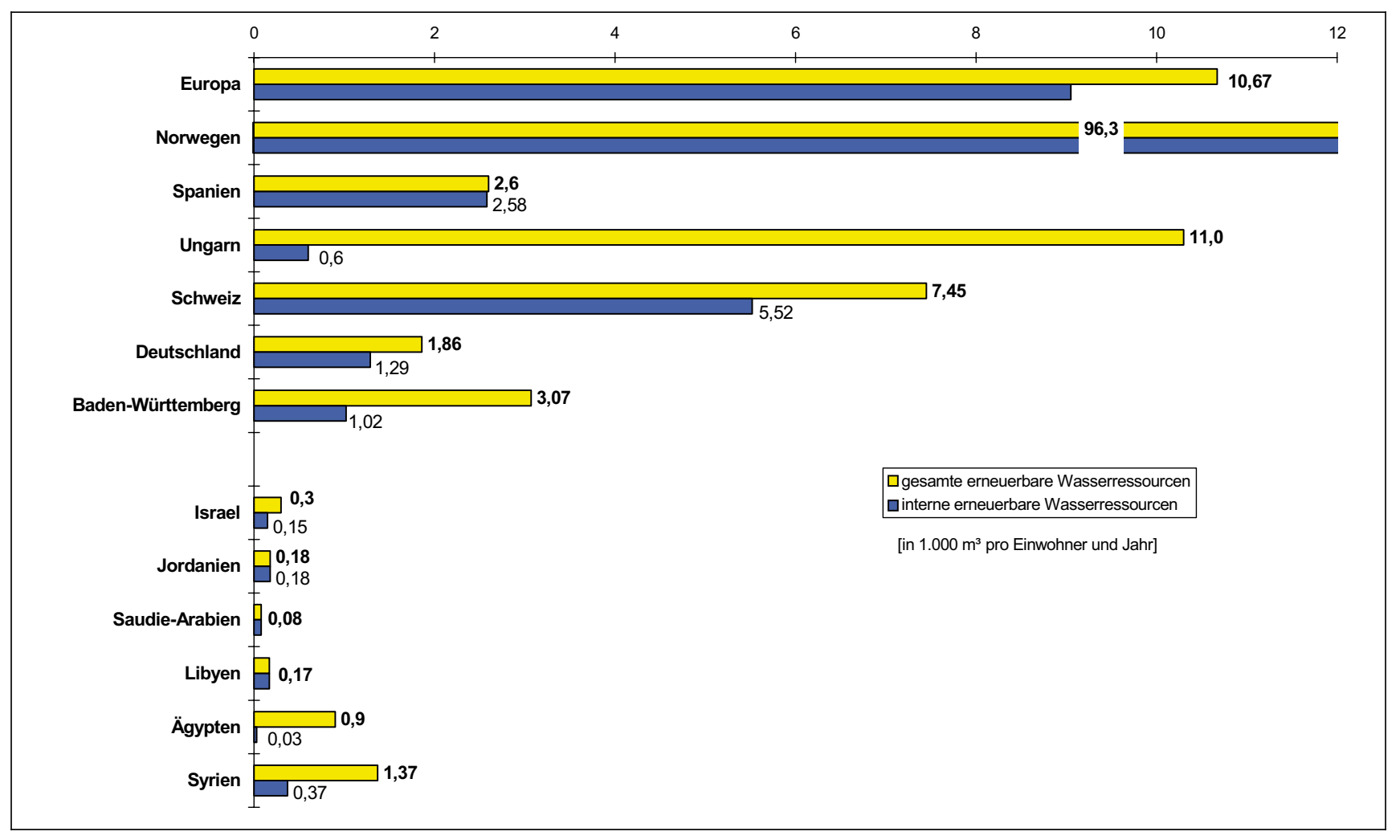

Abb. 2 Erneuerbare Wasserressourcen (in $1.000 \mathrm{~m}^{3}$ pro Einwohner und Jahr) in ausgewählten Regionen (nach Lehn 2002) 
sich auf nationalem Level Unterschiede im spezifischen Wasserdargebot um den Faktor 700 (Libyen zu Norwegen) feststellen.

Ebenfalls deutlich werden in dieser Abbildung die aufgrund naturräumlicher Gegebenheiten bestehenden $\mathrm{Ab}$ hängigkeiten einzelner Staaten von ihren Nachbarn. Durch Vergleich der hellen Balken (gesamte jährliche Wassererneuerungsrate) mit den dunklen Balken (auf nationalem Territorium erneuerter Anteil) - lässt sich der Anteil des Zuflusses und damit die Abhängigkeit von flussaufwärts gelegenen Staaten (Oberliegern), wie z. B. im Falle von Ungarn, Syrien oder Ägypten, deutlich erkennen.

\section{Süßwasser: Verfügbarkeit und Nachfrage}

In einer anthropozentrischen Sicht steht den vorhandenen Wasserressourcen der Bedarf der Menschen gegenüber, die Wasser für unterschiedliche Belange in Anspruch nehmen. Bevor wir diese Inanspruchnahme im zweiten Artikel ausführlicher analysieren, erscheint es sinnvoll, hier zunächst die Verfügbarkeit von Wasser darzustellen und dann exemplarisch eine relevante und aktuell kontrovers diskutierte Nutzungsform aufzuzeigen.

\subsection{Technisch-ökonomische Verfügbarkeit von Wasser}

Die bisherige Darstellung zum Wasserdargebot ließ die Tatsache unberücksichtigt, dass ein Großteil der sich jährlich global erneuernden Wasserressource von ca. $40.000 \mathrm{~km}^{3}$ mit den gegenwärtig zur Verfügung stehenden Techniken nicht ohne Weiteres verfügbar gemacht werden kann. Nach Angaben von Zehnder (2002) fließen nämlich rund zwei Drittel (ca. $27.000 \mathrm{~km}^{3}$ ) bei Starkniederschlägen und Hochwassersituationen in die Ozeane zurück, ohne die Möglichkeit, diese technisch nutzen zu können. Weitere $5.000 \mathrm{~km}^{3}$ regenerieren sich in nur schwer zugänglichen Gegenden, z.B. Sibirien, so dass von den $40.000 \mathrm{~km}^{3}$ lediglich $8.000 \mathrm{~km}^{3}$ heute einer menschlichen Nutzung unmittelbar zugänglich sind. Bezieht man diese Menge auf 6,7 Mrd. Erdbewohner, verbleiben derzeit pro Kopf und Jahr noch $1.200 \mathrm{~m}^{3}$. Im Jahr 2030 verblieben bei $8 \mathrm{Mrd}$. Menschen nur noch $1.000 \mathrm{~m}^{3} / \mathrm{Ea}$ sich jährlich erneuernden Süßwassers. Nach Falkenmark (1989) beschreibt der Bereich zwischen $1.000 \mathrm{~m}^{3} / \mathrm{Ea}$ und $1.700 \mathrm{~m}^{3} / \mathrm{Ea}$ eine Wasser-Stress-Situation für eine Region, bei weniger als $1.000 \mathrm{~m}^{3} /$ Ea beginnt der Bereich des ,absoluten Wassermangels“. Hiernach würde im globalen Mittel bereits heute eine erhebliche Wasser-Stress-Situation vorliegen. In unmittelbarer Zukunft würde globaler Wassermangel drohen.

Da es im Wesentlichen eine Frage der Technik und des Ressourceneinsatzes ist (z.B. für Dammbau und Wasserüberleitungsprojekte), wie viel sich der Mensch von den bisher nicht genutzten rund $32.000 \mathrm{~km}^{3}$ abfließenden Wassers in Zukunft verfügbar machen kann, ist die Frage nach dem „nutzbaren Anteil“ nicht absolut, sondern nur in einem technisch-ökonomischen Kontext zu beantworten. Je nach getroffenen Annahmen variieren dementsprechend die Angaben über die nutzbare Wassermenge. Falkenmark und Rockström z.B. (2004) gehen optimistischer von einem jährlich nutzbaren Anteil von $12.500 \mathrm{~km}^{3}$ aus.

Und selbst der in der Literatur oft zitierte „Basiswert“ von $40.000 \mathrm{~km}^{3}$, der die sich global jährlich erneuernden Wasserressourcen ausweisen soll, ist keine unveränderliche Naturkonstante, sondern eine historisch bedingte und letztlich - im weiten Sinne - dem Stand der Technik geschuldete Größe. ${ }^{6}$ Zusätzliche Techniken, die an anderer Stelle in die globalen Wasserkreisläufe eingreifen, können weitere Wassermengen erschließen. So können z.B. mit Entsalzungstechniken die Salzwassermassen der Ozeane oder über eine Regenwassersammlung auf offener See die ozeanischen Niederschläge erschlossen werden. Zudem ,erschließen" vermehrt zum Einsatz kommende Kreislauftechniken („Wasserrecycling“) zusätzliche Wasserressourcen, nämlich Brauchwasser.

Andererseits ergeben sich durch anthropogene Einflüsse (Umweltverschmutzung) Nutzungsrestriktionen aufgrund unzureichender Qualität „natürlicher“ Wasservorkommen (vgl. Mohrlok und Schiedek 2007; Skutlarek et al. 2006; Ulrich et al. 2002). Gerade in wasserarmen Regionen spielt die Verschmutzung von Oberflächengewässern und Grundwasser eine entscheidende Rolle und trägt - mangels einer adäquaten Aufbereitung - erheblich zur herrschenden Wasserknappheit bei.

$\mathrm{Ob}$ und in welchem Ausmaß knappe Versorgungsverhältnisse bzgl. Wasser herrschen, hängt also nicht allein vom natürlichen Wasserdargebot und der Zahl der zu versorgenden Menschen $a b$, sondern ist - neben den Bedürfnissen und Gewohnheiten dieser - auch stark von den zur Befriedigung dieser Bedürfnisse eingesetzten Techniken abhängig. Letztlich lässt sich die Frage nach der (tatsächlichen) Verfügbarkeit von Wasser nur im konkreten Nutzungskontext beantworten: Welche Wasserressourcen, in welcher Qualität für welche Nutzung unter den Bedingungen welcher Wasserextraktions-, Aufbereitungs- und Transporttechniken verfügbar sind.

\footnotetext{
${ }^{6}$ Er weist die Menge an natürlichen Wasservorkommen aus, die dem Menschen auf ihrem Weg von den Bergen zum Meer bislang unter den (technischen) Bedingungen konventioneller Wassersammel- und -fördertechniken auf dem Festland (zumindest theoretisch) nutzbar war, nämlich das Oberflächen- und Grundwasser. Unter anderen technisch-historischen Bedingungen, bspw. in einer möglichen Zukunft mit Wassergewinnungstechniken, die mengenreich an anderer Stelle in den globalen Wasserkreislauf eingreifen, wie z. B. mit Meerwasserentsalzungsanlagen oder Gewinnung von Wasser aus der Atmosphäre, verliert der Wert von $40.000 \mathrm{~km}^{3}$ als Basiswert der menschlichen Ressource Wasser Sinn und Berechtigung.
} 
Bislang wurde Hub, Reinigung und Transport von Wasser großteils den Kräften der Natur überlassen. Je mehr man diese durch Technik substituiert und zu kontrollieren sucht, desto arbeits-, energie- und ressourcenintensiver wird die Bewirtschaftung von Wasser. Die Verfügbarkeit von Wasser wird so mit fortschreitendem technischem Vermögen (Meerwasserentsalzung, Fernwassertransport, Kreislaufwirtschaft, Wasseraufbereitung, etc.) aus technischer Sicht immer mehr zu einer Frage des Energieeinsatzes, bzw. des Ressourceneinsatzes allgemein.

Im Kontext bereits bestehender oder noch zu entwickelnder Wassertechnologien darf nicht vergessen werden, dass Eingriffe in den natürlichen Wasserkreislauf stets auch Auswirkungen auf die betroffenen Ökosysteme zeitigen. In erster Näherung gilt: Je intensiver die Inanspruchnahme und Kontrolle von Wasser durch den Menschen erfolgt, desto mehr bzw. größere Folgen (insbesondere nicht intendierte Nebenfolgen) sind für Bio-, Geosphäre und Klima zu erwarten.

\subsection{Wasserbedarf bei der Erzeugung von Biomasse}

Betrachtet man sich die Nachfrageseite, so nimmt die Erzeugung von Biomasse zur Verwendung als Nahrungsmittel, Futtermittel, Produktionsstoff oder Energieträger eine herausragende Stellung ein. Weltweit werden über zwei Drittel des geförderten Wassers zur landwirtschaftlichen Bewässerung verwendet. Dieser hohe Anteil ist im Wesentlichen nicht auf uneffiziente Bewässerungstechniken zurück zu führen (obwohl in diesem Bereich durchaus erhebliche Einsparpotenziale zu realisieren sind) sondern darauf, dass der pflanzliche Stoffwechsel selbst sehr wasserintensiv erfolgt. Je nach Pflanzenart werden zur Erzeugung eines Kilogramms Trockenmasse zwischen 250 Liter Wasser (für Sorghum) und 900 Liter (für Alfalfa) benötigt. Weizen nimmt mit 500 Liter Wasser für ein Kilogramm Trockenmasse hier eine Zwischenstellung ein (Muller 1974). Berücksichtigt man weiterhin die nicht verzehrbaren Pflanzenanteile, die in etwa $50 \%$ der Gesamtmasse ausmachen, so bedarf die Erzeugung eines Kilogramms Weizenkörner etwa 1.000 Liter Wasser. Zur Erzeugung eines Kilogramms Reis werden in den Tropen nach Zehnder (2002) bis zu 5.000 Liter Wasser benötigt. Falkenmark und Rockström (2004) nennen eine Spannbreite zwischen 1.150 und 1.900 Liter.

Im Falle tierischer Nahrungsmittel verschlechtert sich diese Bilanz noch einmal erheblich (vgl. Tabelle 2), da Tiere - je nach Art - nur 5 bis 15\% ihres Futters (kalorisch) in Fleisch umsetzen. So bedarf die Produktion eines Kilogramms Rindfleischs z. B. 5.000 (Zehnder 2002) bis 15.000 Liter Wasser (Clarke und King 2004).

Veranschlagt man Ernte- und Produktionsverluste mit $30 \%$ (SEAC 2007), ergibt sich für einen vegetarischen Europäer eine minimale Inanspruchnahme von Wasser
Tabelle 2 Minimale (rechnerische) Pro-Kopf-Wasserbilanz eines Europäers für seine häusliche, öffentliche, gewerbliche Wasserinanspruchnahme sowie zur Erzeugung seiner Nahrungsmittel (3.400 kcal/d); (Daten verändert nach Zehnder 2002 und SEAC 2007)

\begin{tabular}{lcc}
\hline & $\begin{array}{c}\text { Fleischlose } \\
\text { Ernährung }\end{array}$ & $\begin{array}{l}\text { Ernährung } \\
\text { mit 20\% } \\
\text { Fleischanteil }\end{array}$ \\
\hline $\begin{array}{l}\text { Trinken, Hygiene und Haushalt } \\
\text { Öffentliche Dienstleistungen } \\
\text { (Schule, Krankenhaus, etc.) }\end{array}$ & 57 & 57 \\
Industrie (ohne Kraftwerke) & 140 & 35 \\
Teilsumme I & 232 & 140 \\
Fleischlose Nahrungsmittel & 310 & 232 \\
20\% Fleischanteil & 0 & 248 \\
Produktionsverluste 30\% & 93 & 620 \\
Teilsumme II & 403 & 260 \\
& & 1128 \\
SUMME insg. & 635 & 1360 \\
\hline
\end{tabular}

von $635 \mathrm{~m}^{3} / \mathrm{a}$, für sein Fleisch verzehrendes Pendant von $1.360 \mathrm{~m}^{3} / \mathrm{a}$. Nach Falkenmark und Rockström (2004) sind im globalen Durchschnitt $1.600 \mathrm{~m}^{3} / \mathrm{Ea}$ für eine ausbalancierte Ernährung erforderlich. Beide Autoren zitieren frühere Arbeiten (Gleick 2000; Rockström et al. 1999), die für die Realität der 1990er-Jahre etwas geringere Realwerte von etwa 1.200 Liter Wasser pro Einwohner und Jahr im weltweiten Durchschnitt allein zur Erzeugung der Nahrungsmittel benennen. Entsprechend der absoluten Aufnahme kalorischer Energie aus Nahrungsmitteln und abhängig vom Fleischanteil schwankt der tatsächliche Wasserbedarf zur Bereitstellung der Nahrungsmittel weltweit zwischen 600 und $900 \mathrm{~m}^{3} / \mathrm{Ea}$ (in Afrika) und 1.700 bis $1.800 \mathrm{~m}^{3} / \mathrm{Ea}$ (in Europa und Nordamerika) (Gleick 2000). Eine Umstellung der Ernährungsgewohnheiten würde also - zumindest für die industrialisierten Länder - sehr viel mehr Wassersparpotenzial mobilisieren als dies sämtliche Wassersparmaßnahmen im Haushalt vermögen.

Die weitaus größte Wassermenge pro Einwohner (ca. $80 \%$ ) wird somit zur Erzeugung von Nahrungsmitteln benötigt. In Abhängigkeit von der Kalorienzahl der Nahrung, ihrem Fleischanteil und den klimatischen Aufwuchsbedingungen sind insgesamt 1.000 bis $2.000 \mathrm{~m}^{3}$ Wasser pro Einwohner und Jahr erforderlich (Gleick 2000; Falkenmark und Rockström 2004). Rockström und Kollegen (1999) schätzten für die Mitte der 1990er-Jahre den globalen Durchschnittswert zur Erzeugung von Nahrungsmitteln auf $1.200 \mathrm{~m}^{3} / \mathrm{Ea}$. Bei einer von der Food and Agriculture Organization [FAO] für das Jahr 2030 in industrialisierten Ländern prognostizierten Kalorienzufuhr von $3.500 \mathrm{kcal} / \mathrm{Ed}$ errechnet der Schwedische Umweltbeirat der Regierung unter der Annahme eines $40 \%$ Fleischanteils einen jährlichen Wasserbedarf von $2.430 \mathrm{~m}^{3}$ - ohne Produktionsverluste 
(SEAC 2007). Hierzu addieren sich für Haushalt, Industrie und Dienstleistungen weitere $232 \mathrm{~m}^{3}$ (Europa) bis $366 \mathrm{~m}^{3}$ (USA) pro Einwohner und Jahr (Zehnder 2002). ${ }^{7}$

Zusätzliche Brisanz erhält die wasserintensive Erzeugung von Biomasse durch den Trend, dass diese weltweit nicht nur als Nahrungs-, Futtermittel und als Rohstoff, sondern rapide zunehmend auch als Energieträger verwendet wird. Dieser Boom der Bioenergie birgt zum einen Konfliktpotenzial in der künftigen Flächennutzung (Erzeugung von Lebensmitteln oder Energiepflanzen) und dürfte zum anderen einem nachhaltigen Wassermanagement quantitativ (erhöhte Wassernutzung) und qualitativ (mehr Dünger und Pestizide) zunächst einmal entgegenstehen. In seinen Szenarien für das Jahr 2050 geht der Schwedische Umweltbeirat von einer zusätzlichen Evapotranspiration der Energiepflanzen je nach Pflanze und Anbauregion von 3.900 bis 11.750 Mrd. $\mathrm{m}^{3}$ und dementsprechend von einem zusätzli-

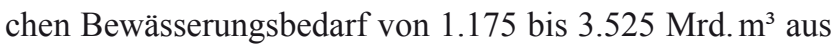
(SEAC 2007). Der Agrarsektor stellt nicht zuletzt deshalb einen wesentlichen Schlüssel zum strategischen Umgang mit der Ressource Wasser dar.

\section{Kritik und Ausblick}

Unser Fazit mündet zunächst in eine Kritik der gängigen Sicht auf Wasser als Ressource und eröffnet anschließend einen Ausblick für die im zweiten und dritten Beitragsteil folgenden Betrachtungen zur Wassernutzung und zum nachhaltigen Umgang mit der Ressource Wasser.

Ein zentraler Kritikpunkt lautet, dass die üblichen Mengenangaben über die (global, kontinental oder regional) zur Verfügung stehenden Wasserressourcen oft mangelhaft begründet und inadäquat gewählt werden. Unserer Ansicht nach ist die Angabe zur Menge des sich jährlich global erneuernden Süßwasservorkommens im Hinblick auf die gesamte menschliche Wassernutzung deutlich zu korrigieren. Die in der heutigen Literatur dominierende Angabe von etwa $40.000 \mathrm{~km}^{3}$ (vgl. Abschn. 2.1) ist auf über $100.000 \mathrm{~km}^{3}$ anzuheben. ${ }^{8}$

Dem Menschen stehen potenziell (und in weiten Teilen eben auch tatsächlich) nicht nur die vom Festland zum Meer zurückfließenden $40.000 \mathrm{~km}^{3}$ zur Verfügung, sondern eben auch die zusätzlich von Land evapotranspirierten $71.000 \mathrm{~km}^{3}$. Das ist umso mehr von Bedeutung, als die terrestrische Evapotranspiration mit $71.000 \mathrm{~km}^{3}$ Wasser jährlich mehr zum Niederschlag über Land - und damit zur Erneuerung der Ressource - beiträgt als die vom Meer herkommenden $40.000 \mathrm{~km}^{3}$ (vgl. Abb. 1).

\footnotetext{
${ }^{7}$ Ohne Wasserbedarf zur Energieerzeugung

${ }^{8}$ Folgt man den Mengenangaben aus Abb. 1 (Lemke 2002), dann ergeben sich rein rechnerisch $111.000 \mathrm{~km}^{3}$.
}

Damit gerät auch die zur Aufzucht von Pflanzen benötigte Wassermenge - gleich, ob sie aus künstlicher Bewässerung oder aus Regenspende stammt - in den Blick (Tabelle 3). Aussagekräftige Dargebot-Nachfrage-Bilanzen sollten bezüglich der Verfügbarkeit von Wasserressourcen insbesondere das über die Pflanzen stetig transpirierte ,grüne" Wasser berücksichtigen, das mit $65.000 \mathrm{~km}^{3}$ mehr als $90 \%$ der von Land evapotranspierierten Wassermenge ausmacht. Das, was aus Sicht der Hydrologie als „Transpirati-

Tabelle 3 Abschätzung von Wasserentnahmen bzw. Wassernutzungen von Fließwegen blauen und grünen Wassers, um direkte und indirekte von Wasser abhängige menschliche Aktivitäten aufrecht zu erhalten (Falkenmark und Rockström 2003

\begin{tabular}{|c|c|c|c|c|}
\hline & Wassertyp & Nutzung & $\begin{array}{l}\text { Wasser- } \\
\text { menge } \\
\left(\mathrm{km}^{3} / \mathbf{a}\right)\end{array}$ & $\begin{array}{l}\% \text { des } \\
\text { Nieder- } \\
\text { schlags }\end{array}$ \\
\hline A & Blau (direkt) & $\begin{array}{l}\text { Landwirtschaftli- } \\
\text { che Bewässerung }\end{array}$ & $1.800^{\mathrm{a}}$ & 2 \\
\hline B & Blau (direkt) & $\begin{array}{l}\text { Haushalt und } \\
\text { Industrie }\end{array}$ & $1.300^{\mathrm{a}}$ & 1 \\
\hline $\mathrm{C}$ & Blau (indirekt) & $\begin{array}{l}\text { „Instream eco- } \\
\text { logy“ }\end{array}$ & $9.400^{b}$ & 8 \\
\hline \multirow[t]{2}{*}{$\mathrm{D}$} & & $\begin{array}{l}\text { Hochwasserab- } \\
\text { fluss }\end{array}$ & $30.150^{c}$ & 27 \\
\hline & $\begin{array}{l}\text { Teilsumme } \\
\text { „Blaues Wasser“ }\end{array}$ & & 42.650 & 38 \\
\hline $\mathrm{E}$ & Grün (direkt) & Nahrungsmittel & $5.000^{d}$ & 4 \\
\hline $\mathrm{F}$ & & $\begin{array}{l}\text { Permanentes } \\
\text { Weideland }\end{array}$ & $20.400^{\mathrm{e}}$ & 18 \\
\hline G & Grün (indirekt) & $\begin{array}{l}\text { Grünland } \\
\text { („Grasslands“) }\end{array}$ & $12.100^{\mathrm{f}}$ & 11 \\
\hline $\mathrm{H}$ & & Forst und Wald & $19.700^{f}$ & 17 \\
\hline I & & Trockengebiete & $5.700^{\mathrm{f}}$ & 5 \\
\hline K & & Feuchtgebiete & $1.400^{\mathrm{f}}$ & 1 \\
\hline $\mathrm{L}$ & & $\begin{array}{l}\text { Verdunstung von } \\
\text { Seen }\end{array}$ & 600 & 1 \\
\hline M & & $\begin{array}{l}\text { Verdunstung von } \\
\text { Stauseen }\end{array}$ & $160^{\mathrm{f}}$ & 0,1 \\
\hline $\mathrm{N}$ & & $\begin{array}{l}\text { Städtische Grün- } \\
\text { flächen }\end{array}$ & $100^{\mathrm{g}}$ & 0,1 \\
\hline \multirow[t]{3}{*}{$\mathrm{O}$} & & $\begin{array}{l}\text { Sonstiges grünes } \\
\text { Wasser }\end{array}$ & 5.690 & 5 \\
\hline & $\begin{array}{l}\text { Teilsumme } \\
\text { „,Grünes Wasser“ }\end{array}$ & & 70.850 & 62 \\
\hline & SUMME insg. & & 113.500 & 100 \\
\hline
\end{tabular}

a) Schätzungen für das Jahr 2000 (Shiklomanov 2000)

b) Nach Postel (1998) beträgt der nutzbare Abfluss $12.500 \mathrm{~km}^{3} / \mathrm{a}$. $12.500-(\mathrm{A}+\mathrm{B})=\mathrm{C}$

c) Ungenutzt ins Meer

d) Rockström et al. (1999) schätzen den kompletten Wasserbedarf zur Nahrungsmittelerzeugung im Schnitt der Jahre 1992-1996 auf $6.800 \mathrm{~km}^{3}$. Hiervon A abgezogen ergibt E

e) 34 mio. $\mathrm{km}^{2}$ (FAOSTAT 1999) mal einer durchschnittlichen Evapotranspiration von $600 \mathrm{~mm}$

f) Nach Rockström et al. 1999

g) L'vovich and White 1990; Rockström et al. 1999 
onsverlust" der Pflanzen bezeichnet wird, ist wesentlicher Bestandteil der Regeneration von Süßwasserressourcen. ${ }^{9}$

Geht man nun von einer jährlich verfügbaren, im globalen Wasserkreislauf natürlich „regenerierten“ Süßwassermenge von (rechnerisch) $111.000 \mathrm{~km}^{3}$ aus, dann bedeutet dies, dass bei der heutigen Bevölkerung jedem Erdenbürger ca. $17.000 \mathrm{~m}^{3}$ pro Jahr zur Verfügung stehen. Bei 8,2 Mrd. Menschen im Jahr 2030 wären dies immer noch knapp $14.000 \mathrm{~m}^{3}$ pro Einwohner und Jahr. Dies liegt weit über dem Schwellenwert des „Wasserstresses“ von $1.700 \mathrm{~m}^{3} / \mathrm{Ea}$ (Falkenmark 1989).

Man beachte, dass in diesen Zahlen zur Verfügbarkeit weder Techniken zur Gewinnung von Frischwasser durch Meerwasserentsalzung, die momentan große Fortschritte machen und immer stärker zur Anwendung kommen (vgl. Grunwald et al. 2008), noch denkbare (einfache) Techniken, wie das Sammeln von Regenwasser auf offener See, berücksichtigt sind. Durch diese Techniken könnten weitere große, natürliche Wasserreservoire (Ozeane, Niederschlag über Ozeanen; vgl. Abb. 1) erschlossen, und die Verfügbarkeit von Nutzwasser weiter erhöht werden.

In der globalen Perspektive und letztlich auch in der kontinentalen (vgl. Tabelle 1) lässt sich auf absehbare Zeit bezüglich der Ressource Wasser kein Mangel erkennen. Es gibt keine globale Wasserkrise. Das Problem der Menschheit besteht nicht darin, dass sich global zu wenig Süßwasser regeneriert, sondern dass sich örtliche und zeitliche Verfügbarkeiten der vorhandenen Wasserressourcen oftmals nicht mit den Bedürfnissen der Menschen decken.

Dennoch lassen sich de facto regional und lokal vehement Wasserkrisen und Wasserstress ausmachen. Wassermangel (wie auch Wasserüberschuss) sind also auch heute noch - geographisch gesehen - regionale oder lokale Phänomene und müssen vor allem von dieser Ebene ausgehend gelöst werden. Auf Basis einer umfassenden und angemessenen Dargebots-Nachfrage-Bilanzierung, die alle Wassernutzungsformen integriert, sollten nationale und internationale Potenziale zur Deckung der unterschiedlichen Süßwasserbedarfe analysiert werden. Darauf aufbauend wären angepasste Versorgungsszenarien zu entwickeln, die auch den Handel mit so genanntem virtuellem Wasser umfassen (siehe Teil II).

Weiterhin lässt sich sagen, dass die Versorgung der Menschen mit Wasser heutzutage kein im Kern technisches Problem darstellt. Es stehen mannigfaltige Techniken zur Verfügung - seien es Hightech- oder Lowtech-Lösungen - die Extraktion, Fassung, Leitung und Speicherung von Wasser (also dessen räumliche und zeitliche Verfügbarkeit) gewähr-

\footnotetext{
${ }^{9}$ Die beim Transpirationsvorgang erforderliche Passage des Wassers durch die Membranen der Pflanzenzellen kann unter Gesichtspunkten der Wasseraufbereitung durchaus als biologische Membranfiltration aufgefasst werden.
}

leisten können (vgl. Jörissen et al. 2008). Die Versorgung aller heute lebenden Menschen mit ausreichend Wasser ist somit vornehmlich kein (ingenieur-)technisches Problem, sondern vielmehr ein politisches. Auf globaler Ebene stellt sich Wassermangel nicht als Problem des (technischen) Könnens, denn vielmehr als Problem des (politischen) Erkennens und Wollens dar (s. Teil III).

\section{Literatur}

Borrazzo J, Rainey R (2007) What Role should Household Drinking Water Treatment and Safe Storage Play in Water Supply Activities and in Health Activities? In: Progress and Prospects on Water. Striving for Sustainability in a Changing World. Abstract Volume of World Water Week. Stockholm International Water Institute, Stockholm, pp 58-59

Clarke R, King J (2004) The Atlas of Water. Mapping the World's most critical Resource. Earthscan, London

Crosa C, Stefani F, Bianchi C, Fumagalli A (2006) Water Security in Uzbekistan: Implication of Return Waters on the Amu Darya Water Quality. ESPR 13(1):37-42

Dawoud K (2001) Ägyptens sensibler Lebensquell. UNESCO Kurier 9:54-55

Deutsche Stiftung Weltbevölkerung (2008) http://www.dsw-online.de/ cgi-bin/count.pl (21.9.2008)

Dyck S, Peschke G (1983) Grundlagen der Hydrologie. VEB Verlag für Bauwesen, Berlin

Falkenmark M (1989) The Massive Water Scarcity Now Threatening Afrika - Why isn't being Addressed? Ambio 18:112-118

Falkenmark M, Rockström J (2004) Balancing Water for Humans and Nature. The new approach in Ecohydrology. Earthscan, London

FAOSTAT - UN Food and Agriculture Organisation Statistical Database (1999) http://faostat.fao.org

Froebrich J, Bauer M, Ikramova M, Olsson O (2007) Water Quantity and Quality Dynamics of the THC - Tuyamuyun Hydroengineering Complex - and Implications for Reservoir Operation. ESPR 14(6):435-442

Gleick PH (2000) The World's Water 2000-2001. Covelo, Washington D.C.

Grunwald A, Schippl J, Dieckhoff C, Gronwald N, Hartlieb N, Jörissen J, Mielicke U, Parodi O, Reinhardt T, Stelzer V (2008) Roadmap Umwelttechnologien 2020 - Zwischenbericht. http://www. itas.fzk.de/deu/lit/2008/scua08a.pdf

HAW - Heidelberger Akademie der Wissenschaften (2007) Wasser - Konfliktstoff des 21. Jahrhunderts? http://www.wasser konferenz.de

Jörissen J, Schippl J, Dieckhoff C, Gronwald N, Grunwald A, Hartlieb N, Mielicke U, Parodi O, Reinhardt T, Stelzer V (2008) Roadmap Umwelttechnologien 2020 - State-of-the-Art-Report (Kurzfassung). Wissenschaftliche Berichte FZKA 7425. Karlsruhe

Kern D, Lorenz WG (2008) Rückstände ausgewählter Humanarzneimittel in Oberflächenwasserkörpern. UWSF 20(2):97-101

Kratz W, Abbas B, Link I (2000) Arzneimittelwirkstoffe in der Umwelt. UWSF 12(6):343-349

Lehn H (2002) Ist unsere Siedlungsentwässerung noch zeitgemäß? In: Parthier B (Hrsg) Wasser - essentielle Ressource und Lebensraum. Nova Acta Leopoldina NF 85(323):347-374

Lehn H, Steiner M, Mohr H (1996) Wasser, die elementare Ressource - Leitlinien einer nachhaltigen Nutzung. Springer, Berlin

Lemke P (2002) Ozeane als Klimaregulatoren. In: Parthier B (Hrsg) Wasser - essentielle Ressource und Lebensraum. Nova Acta Leopoldina NF 85(323):149-175 
Lozán JL, Graß1 H, Hupfer P, Menzel L, Schönwiese C-D (Hrsg) (2005) Warnsignal Klima: Genug Wasser für alle? Wissenschaftliche Auswertungen, Hamburg

L'vovich MI, White GF (1990) World Water Resources and their future (übersetzt von der Amerikanischen Geophysikalischen Union). LithoCraftersInc, Chelsea.Michigan

Mohrlok U, Schiedek T (2007) Urban Impact on Soils and Groundwater - From Infiltration Processes to Integrated Urban Water Management. JSS 7(2):68

Muller WH (1974) Botany: A functional approach. 3. ed. Macmillan, New York

Postel SL (1998) Water for Food Production: Will There Be Enough in 2025? BioScience 48(8):629-637

Püttmann W, Keil F, Oehlmann J, Schulte-Oehlmann U (2008) Wassertechnische Strategien zur Reduzierung der Trinkwasserbelastung durch Arzneimittelwirkstoffe. UWSF 20(3):209-226

Rockström J, Gordon L, Folke C, Falkenmark M, Engwall M (1999) Linkages among water vapor flows, food production and terrestrial ecosystem services. Conservation Ecology 3(2):5. http://www. ecologyandsociety.org/vol3/iss2/art5/

SEAC - Swedish Environmental Advisory Council (2007) Scenarios on economic growth and resource demand. Västra Aros, Stockholm, http://www.sweden.gov.se/content/1/c6/08/04/36/13c3422b.pdf

Shiklomanov IA (2000) Appraisal and assessment of world water resources. Water International 25(1):11-32

Skutlarek D, Exner M, Färber H (2006) Perfluorierte Tenside (PFT) in der aquatischen Umwelt und im Trinkwasser. UWSF 18(3):151-154
Stock M (2003) Klimafolgenforschung: Mögliche Auswirkungen von Klimaänderungen auf die Gesellschaft - Problemstellung und Grundlagen. UWSF 15(4):251-261

Süddeutsche Zeitung (2007) Kriegsgrund Wasser? http://www.sueddeutsche.de/wissen/artikel/107/59048/ (21.3.2007)

Ulrich M, Schulze T, Leist E, Glaß B, Maier M, Maier D, Braunbeck T, Hollert H (2002) Ökotoxikologische Untersuchung von Sedimenten und Schwebstoffen - Abschätzung des Gefährdungspotenzials für Trinkwasser und Korrelation verschiedener Expositionspfade (Acetonischer Extrakt, Natives Sediment) im Bakterienkontakttest und Fischeitest. UWSF 14(3):132-137

UNESCO (2007) Internationale Dekade „Water for Life“, http://www. unesco.de/wasserdekade.html

Völpel E (2008) Schönreden und Einsperren - Der widersprüchliche Umgang der Chilenischen Regierung mit den Mapuche. Lateinamerikanachrichten 408:23-25

Wittfogel KA (1962) Die orientalische Despotie. Eine vergleichende Untersuchung totaler Macht. Kiepenheuer \& Witsch, Köln

Wolf A (2001) Säbelrasseln unter durstigen Anrainern. Interview von Aaron Wolf durch Atmy Otchet. UNESCO Kurier 9:42-43

World Resources Institute (2007) http://earthtrends.wri.org/searchable_db/index.php?step $=$ countries \&ccID $\% 5 B \% 5 \mathrm{D}=0$ \& theme $=2$ \&variable_ID $=1 \&$ action $=$ select_years

Zehnder JB (2002) Wasserressourcen und Bevölkerungsentwicklung. In: Barthier B (Hrsg) Wasser - essentielle Ressource und Lebensraum. Nova Acta Leopoldina NF 85(323):399-418 